\title{
Avis d'une mère à sa fille: la traducción al español de la condesa de Lalaing
}

\section{Advice of a mother to her daughter: translated into Spanish by the Comtesse of Lalaing}

\author{
María del Carmen Aguilar Camacho \\ I52agcam@uco.es
} Universidad de Córdoba

Fecha de recepción: 16 de marzo de 2017

Fecha de aceptación: 15 de mayo de 2017

Resumen: en este trabajo se lleva a cabo un análisis traductológico de uno de los tratados de educación más celebrados de Mme de Lambert. En él, aconseja a su hija cómo comportarse para conseguir el respeto de los demás conduciéndose de forma virtuosa. En primer lugar, y previo al análisis traductológico, se aborda el contexto histórico-social de la autora así como las influencias de los grandes pensadores clásicos y coetáneos en los que se inspiró la autora para escribir su obra. Finalmente, se aborda el análisis traductológico.

Palabras clave: análisis traductológico, traducción literaria, técnicas de traducción, errores de traducción y salones literarios

Abstract: this paper deals with a translatological analysis of one of Mme de Lambert's most important treatise on education. In this tract, she advises her daughter how to behave to gain the respect of others leading a virtuous life. First of all and previous to the analysis, we study the historical-social context of this salonnière and the influences of the great classic and coetaneous philosophers and thinkers' influences on her work. Finally, we tackle the translatological analysis.

Key words: Translatological analysis, literary translation, translation techniques, translation errors y literary salons. 


\section{LA MARQUESA DE LAMBERT Y EL HÔTEL DE NEVERS}

Anne-Thérèse de Marguenat de Courcelles, Marquesa de Lambert y anfitriona del célebre Hôtel de Nevers, nace en París en 1647 y muere en 1733. Debido al momento histórico en el que vive así como la educación recibida, en su figura, se perfilan rasgos propios tanto del Clasicismo como de la Ilustración. François Le Coigneux señor de la Rocheturpin et de Bachaumont ${ }^{1}$, literato diletante conocido en círculos libertinos y segundo marido de la madre de la marquesa, ejerce sobre ella una notable influencia al observar su capacidad y disposición por aprender. Efectivamente, Mme de Lambert rechaza, al contrario que sus hermanas, la idea de formar parte de una congregación religiosa y decide permanecer en la casa familiar y tratar con los poetas epicúreos, señores libertinos y la élite de la sociedad parisina de la época que la frecuentaban. Bachaumont le infundió su amor por la literatura y el gusto por los Clásicos y le proporcionó una cuidada educación basada en el escepticismo. También le muestra que no es necesario ceñirse a un único planteamiento, sino que puede combinar los placeres frívolos con los pensamientos más profundos.

II s'affectionna à sa Belle-Fille, presque encore enfant, à cause des dispositions heureuses qu'il découvrit bien-tôt en elle, \& il s'appliqua à les cultiver, tant par lui-même que par le monde choisi qui venoit dans sa Maison, \& dont elle apprenoit sa Langue comme on fait la Langue maternelle. (Marguenat de Courcelles, 1747: XII-XIII).

En torno a 1710, madame de Lambert abre las puertas de su salón dos días en semana (martes y miércoles) para recibir tanto a ilustres personajes aristocráticos como a importantes literatos ${ }^{2}$. La anfitriona del salón de Nevers huye de los frívolos divertimentos tan del gusto de la época y exige que sus invitados sean exponentes del ideal del honnête homme ${ }^{3}$ y honnête femme. Entre los asiduos a su hotel podemos mencionar a Houdar la Motte y Anne Dacier (que mantuvieron posturas enfrentadas en la denominada Querelle des Anciens et Modernes), Montesquieu, Fontenelle, Abbé de Choisy,

\footnotetext{
${ }^{1}$ Bachaumont escribió junto con su amigo, Claude Emmanuel Louillier, más conocido como monsieur La Chapelle por su lugar de nacimiento, el Voyage de Bachaumont et Chapelle.

${ }^{2}$ Además, fue una de las primeras damas de su época que invitaba a su salón a actores, tales como, Adrienne Lecouvreur (actriz que con posterioridad abriría su propio salón en la Rue de Marais y entre cuyos contertulios podemos contar con la presencia de Bernard le Bovier de Fontenelle, la duquesa du Maine o François Marie Arouet, Voltaire) y Michel Baron (actor amigo de Molière, Corneille y Racine de los que interpretó a algunos sus personajes).

${ }^{3}$ En 1630, Nicolas Faret escribió L'honnête homme ou l'art de plaire à la cour, en esta obra sintetiza las cualidades que deben reunir aquellos que quieran tener éxito en la sociedad. En el honnête homme se aprecia el buen gusto, el respeto por el orden establecido, unos sentimientos nobles, una amplia cultura sin caer en la pedantería, una moral ejemplar y, además, una conversación agradable.
} 
Fénelon, Hénault, Marivaux, Louis de Sacy, le marquis de Sainte-Aulaire ${ }^{4}$, Baronne Staal, Madame de Tencin y le marquis d'Argenson, entre otros.

Además de la altura de sus invitados y de constituir un reducto del buen gusto, de la cultura exquisita y de impecables formas, se puede afirmar que el Hôtel de Nevers está considerado como la antecámara de la Académie française ${ }^{5}$ debido a que no pocos de los candidatos a la misma mostraban allí su talento para concitar el apoyo de la marquesa y de su círculo.

\section{AVIS D'UNE MERE A SA FILLE}

De su matrimonio con Henri de Lambert, marqués de Saint-Bris, nacen sus dos hijos: Henri-François y Marie-Thérèse ${ }^{6}$, a los que escribe respectivamente sus conocidos Avis d'une mère à son fils y Avis d'une mère à sa fille. El primero de ellos aparece publicado en 1726 en Mémoires de Littérature du Père Des Molets, sous le titre de Lettre d'une Dame à son Fils sur la vraie Gloire.

El segundo se publica en 1728 y, aunque desconocemos la fecha exacta en que Madame de Lambert escribió este opúsculo, es generalmente aceptado que fue en 1698.

La edición que se ha utilizado para este trabajo es la de 1747, impresa en Lausanne por Bousquet \& Compagnie y lleva por título CEuvres de Madame la marquise de Lambert, rassemblées pour la premiere fois ${ }^{7}$.

\subsection{Estructura}

La obra comienza con la misma preocupación que le asalta en el Avis dedicado a su hijo:

\footnotetext{
${ }^{4}$ El presidente Hénault en sus memorias escribe: “On croit qu'elle s'était mariée au marquis de Sainte-Aulaire [...]." (Hénault, 1971:120). François-Joseph de Beaupoil, marqués de SaintAulaire, fue miembro de la Académie Française desde 23 de septiembre de 1706, sustituyendo Jacques Testu de Belval. "C'était un homme d'esprit, qui ne s'avisa qu'à plus de soixante ans de ses talents pour la poésie, et que Mme de Lambert, dont la maison était remplie d'académiciens, fit entrer à l'Académie française, non sans assez de résistance de la part de Despréaux et de quelques autres, résistance qui n'était pas fondée." (Hénault, 1971:121)

5 "Il est certain qu'elle avoit bien fait la moitié des académiciens." (Delorme, 1951: 224).

${ }^{6}$ Del matrimonio entre Henry de Lambert y Anne-Thérèse nacen cuatro hijos: Henry-François de Lambert, marqués de Saint-Bris, Marie-Thérèse y otras dos hijas fallecidas a temprana edad. Marie-Thérèse ("[...] qui avoit été mariée en 1703 avec Louïs De Beaupoil, Comte de St. Aulaire, Seigneur de la Porcherie \& de la Grenellerie, Colonel Lieutenant du Régiment d'Enguin, Infanterie, tué au combat de Ramersheim dans la haute Alsace le 26 Août 1709, elle est morte le 13 Juillet 1731 âgée de 52 ans, ayant laissé une fille unique, nommée Thérèse-Eulalie de Beaupoil de St. Aulaire.") es la destinataria de estos consejos. (Marguenat de Courcelles, Anne Thérèse (de): Oeuvres de Madame la marquise de Lambert, rassemblées pour la premiere fois. Lausanne: Bousquet \& Compagnie, 1747, p. XI)

${ }^{7}$ Las citas extraídas de la obra han sido transcritas con total respeto a su grafía original.
} 
Quelques soins que l'on prenne de l'Education des enfans, elle est toujours très imparfaite (Lambert, 1747: 1).

La inquietud por la falta de diligencia en la educación de los jóvenes es una constante en la obra de la marquesa que se pone de manifiesto en las primeras reflexiones vertidas en ambos Avis. El Avis dedicado a su hija comienza con estas palabras:

On a dans tous les tems négligé l'éducation des Filles (1747: 55).

Considera que la educación femenina es négligée, mientras que la masculina es très imparfaite. Deja entrever que la primera, al quedar en manos de ayas procedentes del pueblo llano e imbuidas de supersticiones, es aún más rudimentaria que la segunda. Mme de Lambert culpa de esta falta de atención al papel tradicional que se reserva a la mujer en la sociedad de su época que las aboca a la indigencia intelectual.

On les destine à plaire; on ne leur donne des leçons que pour les agrémens; on les livre à la mollesse, au monde \& aux fausses opinions; on ne leur donne jamais de leçons de vertu ni de force. (1747: 56)

Y es la propia sociedad la que obvia que:

[...] elles composent la moitié du monde, elles font le bonheur ou malheur des hommes, que par elles les maisons s'élevent ou détruisent et que l'éducation des enfants leur est confiée dans la première jeunesse [...]. (1747: 55)

Les Filles naissent avec un désir violent de plaire. Comme elles trouvent fermés les chemins qui conduisent à la Gloire \& à l'Autorité, elles prennent une autre route pour y arriver, \& se dédommager par les agrémens. (1747: 70)

Algunos autores se apoyan en premisas como las anteriores para defender el supuesto feminismo de Mme de Lambert. No obstante, a pesar de mostrarse contraria al papel tradicional de la mujer, la marquesa entiende que hay dos principios a los que una mujer debe "obeïr": la Religión y el Honor. Estos principios, que también son exigibles a los hombres, no les afectan de la misma manera. Las mujeres deben vivir la religión de forma diferente a como deben hacerlo los hombres, para los que la cuestión religiosa se asemeja más a una conveniencia social que a una auténtica devoción arraigada en su interior. A su hija, sin embargo, la alienta a mantener el culto diciéndole que: 
Quel sera le principe de ces sentimens? La Religion, quand elle sera gravée dans notre coeur. Alors toutes les vertus couleront de cette source; tous les devoirs se rangeront chacun dans leur ordre. [...] Nous avons tant d'intérêt à pratiquer la Vertu, que nous ne devons jamais la regarder comme notre ennemie, mais comme la source du bonheur, de la gloire $\&$ de la paix. (1747: 57$)$

La Religion seule calme tout, \& console de tout; en vous unissant à Dieu, elle vous reconcilie avec le Monde \& avec vous-même. (1747: 58)

En cuanto al honor:

Quoique l'Honneur soit l'ouvrage des hommes, rien n'est plus réel que les maux que souffrent ceux qui ont voulu s'y dérober [...] il doit régler votre vie, \& que rien n'est plus contraire au repos, \& ne nous donne une conduite plus incertaine, que de penser d'une façon, \& d'agir d'une autre. (1747: 61 )

Fortifiez donc ce préjugé de l'Honneur, \& que votre délicatesse le porte jusques au scrupule. (1747: 61)

Ninguna mujer puede eludir el control de un doble tribunal. Por un lado, su propia e inevitable consciencia, a la que debe convencer de su honestidad. Por otro lado, el mundo, es decir, la aprobación pública puesto que del desprecio a la propia reputación nace el desprecio a la virtud. En este tema, también encontramos una gran diferencia respecto de las palabras que dedica a su hijo, a quien le dice que solo va a ser juzgado por un único tribunal: él mismo.

A continuación, señala cuáles son las virtudes que deben atesorar las mujeres. En primer lugar, menciona la Honte, que es le plus fidele gardien de la Vertu des Femmes. Acto seguido, resalta la importancia de la Pudeur, que equipara al Valeur de los hombres. Ese pudor es el que le hará rehuir la Renommé. Antes de pasar al siguiente sentimiento, trae a colación las palabras de un autor clásico con el que comparte opinión pero solo en parte:

Les grandes vertus sont pour les Hommes; il ne donne aux Femmes que le seul mérite d'être inconnues. Ce ne sont pas celles, dit - il, qu'on loue le plus, qui sont les mieux louées, mais celles dont on ne parle point. La pensée me paroît fausse; mais pour réduire cette maxime en conduite, je crois qu'il faut éviter le monde \& l'éclat, qui prenne toujours sur la pudeur, \& se contenter d'être à soi-même son propre spectateur. (1747: 64)

La Chasteté y la Modestie enaltecen a quienes las ejercitan pues pugnan contra la soberbia y las pasiones vivas y embellecen a la persona, 
incluso a aquella carente de belleza natural. La Beauté y la Jeunesse, cualidades muy apreciadas y deseadas por la mujer, son, sin embargo, efímeras y ejercen una tiranía que engaña a quien las posee, pues las aprovecha para dar rienda suelta a sus pasiones e, indefectiblemente, las aparta de su deber.

Rien n'est plus court, que le regne de la beauté: rien n'est plus triste, que la suite de la vie des Femmes qui n'ont su qu'être belles. (1747:68)

Por ello, aconseja no frecuentar los espectáculos donde las jóvenes suelen acudir para lucirse.

Soyez retenue sur les spectacles. II n'y a point de dignité à se montrer toujours. (1747: 71)

Junto con las virtudes, señala Mme de Lambert, los perniciosos vicios que se deben corregir. Entre ellos menciona la coquetería, la mentira, la vanidad, la voluptuosidad y el amor propio. Como este último no nos permite percatarnos de nuestros defectos, es ineludible el recurso a la equidad, la prudencia y la moral.

Si bien es verdad que hay que aspirar a conducirse de forma que se alcance la virtud, también es cierto que esta la encontramos en cualquier faceta de la vida. Por ello, no se olvida de recomendar ciertos placeres a las mujeres que proporcionarán una alegría más grande que los placeres vivos. Con aquellos placeres, entre los que menciona la lectura, las labores y la conversación, también pueden mostrarse virtuosas. Por el contrario, de los placeres vivos dice:

Quand vous ne vivez que pour les plaisirs [...] l'Ame tombe dans un grand vuide. (1747: 72)

La marquesa revela a su hija que en la edad madura, en la que ella se encuentra y donde la belleza poco a poco se esfuma, solo encontrará calma en la razón, la verdad y la virtud, si es que durante los años de juventud se ha conducido según sus dictados.

No olvida la autora la referencia a los estudios en que debe instruirse cualquier joven porque la curiosidad de la edad no debe reprimirse, antes bien considera que debe ser calmada con conocimiento. Es laudable el interés por las denominadas ciencias sólidas, entre las que alude a la Historia griega y romana, la Historia de Francia, la Filosofía; el Latín es recomendable porque es la lengua de la Iglesia y abre las puertas del conocimiento científico, sin embargo, el italiano es censurable porque es la lengua del amor; en cuanto a la lectura, alaba las tragedias de Corneille porque dan lecciones de virtud 
pero desaconseja la poesía y la novela porque encienden la imaginación. No obstante, la previene de las ciencias extraordinarias entre las que incluye a las Matemáticas y la Astronomía. En síntesis, la educación más conveniente debe ser austera y tradicional puesto que es la que mantiene bajo control la imaginación y ahuyenta los peligros del amor.

[...] rien n'est plus opposé au bonheur, qu'une imagination délicate, vive \& trop allumée. (1747: 86)

En cuanto a los deberes de una mujer, establece que esta debe resistirse a los placeres, convertirse en su mejor amiga, huir del gran mundo, recabar recursos, ser modesta, justa, razonable, inocente, fiel, pudorosa, dócil, buscar la soledad, frenar su imaginación y someterla a la verdad, atenerse a sus propios asuntos y a los de su familia y no esperar demasiado de los hombres.

Respecto del amor, distingue dos momentos: les commencemens en los que todo agrada y que enmascara la realidad.

C'est la plus cruelle situation où une personne raisonnable puisse se trouver (1747: 96)

Y la suite cuando el amor turba el alma de la enamorada y dispara su imaginación.

II jette le trouble dans l'ame \& dans le sens; il enleve la fleur de l'innocence; il étonne la Vertu [...] (1747: 97)

Mme de Lambert previene a su hija de los peligros del amor:

Ne soyez point en commerce avec votre imagination: elle vous peindra l'amour avec tous ses charmes. Tout est séduction, illusion, quand il passe par elle; il y a bien à perdre, quand vous la quittez pour venir à la réalité. [...] Ce ne sont pas toujours les fautes qui nous perdent, c'est la maniere de se conduire après les avoir faites. (1747: 98)

Una vez analizadas las virtudes y los vicios de la esfera privada, Mme de Lambert se traslada a la vida en sociedad y muestra a su hija cuáles son los deberes sociales que debe asumir. En primer lugar, afirma que es necesario fortalecer la razón con principios ciertos a los que acudir en caso de debilidad. Concretando un poco más, la anima a ser generosa con los pobres y a cuidar de los otros rechazando el egoísmo y evitando ser demasiado exigente con los demás. Este es el primer deber de la Vie-civile. Del mismo modo debe hacer oídos sordos frente a las calumnias, evitando 
formar una opinión de forma precipitada y basada solo en las apariencias. También la anima a no condenar a los demás sin haber recabado la información precisa. De este comportamiento, nacen gran parte de las enemistades y los odios que tanto daño hacen a la sociedad. Por otro lado, la anima a ser justa, equitativa y honesta, pues la humildad es una gran virtud que nos asiste para soslayar el desprecio y la envidia.

Cuando nos ofendan, conviene que nos alejemos y nos calmemos, pues ese ataque solo merece nuestro desprecio y buscar consuelo en los amigos.

Las buenas formas y la educación son los principios inspiradores de una conducta impecable en público. Frente a estos principios encontramos la adulación, las risas y la admiración. En efecto, Mme de Lambert cree que:

[...] rarement en faisant rire se fait-on estimer. (1747: 113)

Approuvez, mais admirez rarement; l'admiration est le partage des sots. (1747: 114)

Finalmente, da unas recomendaciones en relación a la actitud que se debe mantener ante les Domestiques. Estos merecen un trato bondadoso y humano que implica, por un lado, emplear unas formas correctas aunque sin abandonar cierta firmeza que no obstante no contempla ni el castigo ni las amenazas. Por otro lado, previene a su hija de los halagos que le prodiguen sus criados, pues con frecuencia son fruto del servilismo debido. Justifica este trato en que solo se debe a un capricho de la fortuna el que unos sean criados y otros no.

Rien n'est si bas, que d'être haut à qui vous est soumis. [...] Le service étant établi contre l'égalité naturelle des hommes, il faut l'adoucir. (1747: 115)

\subsection{Influencias}

En los escritos de Mme de Lambert, aparecen continuas referencias a los grandes pensadores clásicos y a otros moralistas franceses de épocas recientes. En cuanto a los clásicos, se percibe la influencia de epicúreos, como Plutarco, Platón y Diógenes Laertes, y de estoicos, como Séneca, Epicteto y Marco Aurelio. Entre sus contemporáneos, destacan Montaigne, Saint Evremond, La Rochefoucauld, Fontenelle, la Bruyère, Malebranche, Fénelon. Estas influencias que aparecen, unas veces, enmascaradas en el texto y, otras veces, citando al autor, han sido argüidas por algunos estudiosos para afirmar que no estamos ante una autora original.

A continuación, relacionamos algunas de estas influencias más notables. 


\subsection{1. Éducation des Filles ${ }^{8}$ de Fenelon}

La influencia de Fenelon en esta obra es evidente pues la propia marquesa, en su Lettre à Mr. l'Archevêque de Cambray, en réponse à celle que ce Prélat avoit écrite à Mr. De Sacy, escribe:

J'ai trouvé dans Télémaque, les préceptes que j'ai donnés à mon Fils \& dans l'Education des Filles, les conseils que j'ai donnés à la mienne. Je n'ai de mérite, que d'avoir su choisir mon Maître \& mes modèles. (Fénelon, 1687: 402)

A continuación, incluyo algunas de estas coincidencias vertidas a lo largo del texto:

Rien n'est plus negligé que l'éducation des filles (Fénelon, 1867: 1)

On a dans tous les tems négligé l'éducation des Filles (Lambert, 1747: 55)

ce premier âge qu'on abandonne à des femmes indiscretes \& quelquefois déreglées, est pourtant celuy où se font les impressions les plus profondes... (Fénelon, 1867: 17)

[...] que l'éducation des enfans leur est confiée dans la première jeunesse, tems où les impressions se sont plus vives \& plus profondes. (Lambert, 1747: 55)

(...) quoy qu'il ne faille pas toûjours menacer sans châtier, de peur de rendre les menaces méprisables. II faut pourtant châtier encore moins qu'on ne menace (...) (Fénelon, 1867: 58)

II ne faut pas aussi toujours menacer sans châtier, de peur de rendre les menaces méprisables : mais il ne faut appeller [sic] l'autorité, que quand la persuasion manque. (Lambert, 1747: 115)

Elle naissent avec un desir violent de plaire. (Fénelon, 1867: 191)

Les Filles naissent avec un desir violent de plaire. (Lambert, 1747: 69)

La beauté $(\ldots)$ ne serve à faire marier avantageusement un fille (Fénelon, 1867: 201)

La beauté a de grands avantages. (Lambert, 1747: 67)

[...] tout ce qui peut faire sentir l'amour, plus il est adoucy \&enveloppé, plus il me paroist dangereux (Fénelon, 1867: 246)

plus il est adouci, plus il est modeste , \& plus il est dangereux. (Lambert, 1747: 82)

${ }^{8}$ Fénelon, François de Salignac de la Mothe, Éducation des filles. Paris: Pierre Aubouin, P. Emery et C. Clousier, 1687. 


\subsubsection{Traité de morale (1684) de Malebranche ${ }^{9}$}

En el Avis d'une mère à sa fille, Mme de Lambert reproduce las palabras de Malebranche pero sin mencionarlo:

Enfin, comme dit un grand-Homme, pour être Chrétien, il faut croire aveuglément; \& pour être sage, il faut voir évidemment. (Lambert, 1747: 84)

\section{En las CEuvres de Malebranche, podemos leer:}

En un mot, pour être fidèle, il faut croire aveuglément, mais pour être philosophe il faut voir évidemment. (Malebranche, 1864: 25)

\subsubsection{La Rochefoucauld \\ Madame de Lambert nombra a M. de la Rochefoucault y reproduce sus palabras:}

M. de la ROCHEFOUCAULT dit, qu'elle vous conduit jusqu'à la fin de la vie par un chemin agréable. (Lambert, 1747: 92)

Más adelante, encontramos el siguiente pasaje:

Examinez votre caractere, \& mettez à profit vos défauts il n'y en a point qui ne tienne à quelques vertus, \& qui ne les favorise. La Morale n'a pas pour objet de détruire la Nature, mais de la perfectionner. Etes vous glorieuse? servez-vous de ce sentiment-là pour vous élever au-dessus des foiblesses de votre sexe, pour éviter les défauts qui humilient. II y a à chaque dérèglement du cœur une peine \& une honte attachées qui vous sollicite à le quitter. Etes-vous timide? tournez cette foiblesse en prudence: qu'elle vous empêche de vous commettre. Etes-vous dissipatrice ? Aimez-vous à donner ? II est aisé de la prodigalité d'en faire de la générosîté. Donnez avec choix \& à propos... (Lambert, 1747: 93)

\section{Según Zimmerman:}

L'origine de ce curieux passage est peut-être la maxime de La Rochefoucauld: "Les vices entrent dans la composition des vertus comme les poisons entrent dans la composition des remèdes; la

\footnotetext{
${ }^{9}$ Malebranche, Nicolas, QEuvres de Malebranche, T.III, Recherche de la vérité. Paris: Entier et Cie, Libraires-Éditeurs, 1871.
} 
prudence les assemble et les tempère; et elle s'en sert utilement dans les maux de la vie". (1917: 59-60)

\subsubsection{Otros autores}

Ocasionalmente, Mme de Lambert transcribe lo que han dicho otros autores citándolos. A continuación, se reproducen algunos ejemplos:

Souvenez-vous du passage d'HORACE: La Volupté marche devant vous, \& nous cache sa suite. (Lambert, 1747: 91)

PLINE en renvoyant à son Ami une Obligation considérable qu'il avoit de son Pere, avec une quittance générale, lui dit: J'ai peu de bien; je suis obligé à beaucoup de dépense; mais je me suis fait un fonds de ma frugalité, \& c'est d'où je tire les services que je rends à mes Amis. (1747: 75)

Vous allez, disoit MONTAIGNE aux jeunes gens, vers la réputation, vers le crédit, \& moi j'en reviens. (1747: 76)

Faites ce que SENEQUE conseilloit à son Ami Lucile: Choisissez, lui disoit-il, parmi les grands-Hommes celui qui vous paroitra le plus respectable: ne faites rien qu'en sa présence: rende-lui compte de toutes vos actions. (1747: 76)

On pardonnoit tout à Agrippine Femme de Germanicus, en faveur de sa chasteté: cette Princesse étoit ambitieuse \& hautaine; mais, dit TACITE, toutes ses passions étoient consacrées chasteté (1747: 63)

Je voudrois aussi de la Morale: à force de lire CICERON, PLINE, \& les autres, on prend du goût pour la Vertu. (1747: 80)

Vous avez recours à SENEQUE \& à EPICTETE. Est-ce à leur raison à vous consoler? (1747: 85)

Otras veces, no menciona al autor, como en los siguientes ejemplos:

La réflexion, dit un Pere de l'Eglise, est l'œil de l'ame, c'est par elle que s'introduisent la lumière \& la vérité. (1747: 89)

Un Ancien disoit, qu'il s'envelopoit du manteau de sa Vertu [...] (1747: 58)

Un Ancien dit, que les grandes vertus sont pour les Hommes; il ne donne aux Femmes que le seul mérite d'être inconnues. Ce ne sont point 
celles, dit-il, qu'on loue le plus, qui sont les mieux louées; mais celles dont on ne parle point. (1747: 64)

Un Ancien dit, que c'est une courte tyrannie, \& le prémier privilege de la nature; que les belles personnes portent sur le front des lettres de recommandation. (1747: 67)

La tempérance, disoit un Ancien, est la meilleure ouvriere de la volupté. (1747: 73)

Rendez-vous compte, dit un Ancien, de toutes vos heures, afin qu'ayant profité du présent, vous ayez moins besoin de l'avenir. (1747: 79)

J'ai appris, disoit un Ancien, à être mon ami, ainsi je ne serai jamais seul. (1747: 88)

Rien n'est pire, dit un Ancien, que la pauvreté dans les richesses, parce que le mal tient à l'ame. (1747: 106)

Un Ancien dit, qu'il faut les regarder comme des Amis malheureux. (1747: 114)

\section{ANÁLISIS TRADUCTOLÓGICO}

El opúsculo de Mme de Lambert es un tratado de educación que pertenece al género ensayístico. En él, la marquesa aconseja a su hija cómo actuar en determinadas ocasiones de la vida cotidiana, y para ello se sirve de lo aprendido en la lectura de los clásicos y de los pensadores de la época y de su propia experiencia vital. La condesa de Lalaing, con el fin de ofrecer un presente a $\mathrm{D}^{\mathrm{a}}$ Luisa de Borbón, Princesa de Asturias, traduce varias obras de la marquesa y, entre ellas, estos Avis. La traducción mantiene el mismo tono que el texto original, es decir, además de respetar el contenido lingüístico de la misma, intenta conservar el estilo y la intencionalidad de la autora. Podemos afirmar que emplea el método literal pues en su traducción se puede observar que reproduce palabra por palabra, sintagma por sintagma, el texto original. Esta reescritura resulta, a veces, muy forzada e incluso incurre en expresiones desacertadas por mantener dicha literalidad. El título, Avis d'une mère à sa fille, es traducido como Advertencias de una madre á su hija ${ }^{10}$.

Para realizar el análisis traductológico tendremos en cuenta en los cuatro niveles tradicionales: morfosintáctico, léxico-semántico, pragmático-

${ }^{10}$ La edición utilizada para este trabajo es: Cerda y Vera, María Cayetana (de la), Condesa de Lalaing: Obras de la Marquesa de Lambert traducidas del Francés. Madrid: Manuel Martín, 1781. Las citas extraídas de la obra han sido transcritas (del mismo modo que en los textos franceses) respetando su grafía original. 
cultural y ortotipográfico, haciendo referencia tanto a las técnicas de traducción empleadas como a los errores cometidos.

$\mathrm{Si}$ atendemos a la frecuencia con la que se aplica cada técnica, comprobamos que las más recurrentes son la articulación, la reforma de oraciones y la transposición; acto seguido, aunque menos frecuentes, la traductora emplea las modulaciones; y, finalmente, la inversión, la explicitación, la compresión lingüística, la equivalencia, la ampliación lingüística y la elisión.

En el plano ortotipográfico, podemos destacar la falta de coincidencia en el empleo de mayúsculas y minúsculas. Ambas, autora y traductora, han aplicado escrupulosamente las reglas de ortografía propias de cada lengua. Así, podemos ver: Monde - mundo, Vertu - virtud, Tribunaux - tribunales, Loix - leyes, Amour - amor.

Por otro lado, la correcta ortografía de las palabras empleadas en la traducción ha sido comprobada en el DRAE de 1780 vigente en aquel momento. Por ello, llama la atención encontrar algunas de ellas escritas de forma diferente. Algunos vocablos pertenecen a la misma familia por lo que al compartir la misma raíz se advierte en ellas el mismo error. Esto es lo que ocurre por ejemplo con "abanzada" y "abanzando" y con "escusa" y "escusar". "Espectáculo", "estiende", "comfesar", "termino", "quexarnos", "sympatia" o "genti" constituyen otra muestra de esas diferencias ortográficas.

Finalmente, hemos hallado la palabra "horada" que entendemos se debe más a una cuestión tipográfica que ortográfica. En efecto, honnête se puede traducir como "honrada" pero no tiene ninguna coincidencia con el verbo "horadar", que según el DRAE de 1780 significa: "Penetrar alguna cosa, agujereándola de parte a parte."

vous vous devez à vous-même le témoignage que vous êtes une honnête personne. (1747: 62)

Tú te debes a ti misma el testimonio de que eres una persona horada: (1781: 59)

Junto a ello, encontramos que, en ocasiones, la traductora decide utilizar el punto y aparte cuando en el texto original hay un punto y seguido, como en el ejemplo siguiente, en el que este fragmento en el texto original forma parte de un párrafo y en la traducción da comienzo a uno nuevo:

La tempérance [...] est la meilleure ouvriere de la volupté (Lambert, 1747: 73)

La moderacion [...] es la que mejor pone en el debido tono los gustos permitidos. (Lalaing, 1781: 71) 
Alors toutes les vertus couleront de cette source; tous les devoirs se rangeront chacun dans leur ordre. (1747: 57)

Entonces todas las virtudes nacerán de este principio, y todas las obligaciones se pondrán en su lugar. (1781: 54)

[...] on lui permit de se mêler avec toutes les autres. Depuis ce tems-là , elle en est inséparable (1747: 66)

[...] la permitió mezclarse con todas las otras; y desde este tiempo es inseparable. (1781: 64)

A quoi se prendre? le passé nous fournit des regrets; le présent, des chagrins; \& l'avenir des craintes. (1747: 58 )

¿A qué se han de dedicar? Si lo pasado las subministra penas, lo presente pesadumbres, y lo futuro temores: [...] (1781: 56)

La propreté est un agrément, \& tient son rang dans l'ordre des choses gracieuses; (1747: 70)

La limpieza es un adorno, que tiene su lugar en el orden de las cosas graciosas; (1781: 68)

Que le faste ne vous impose pas: il n'y a que les petites âmes qui se prosternent devant la Grandeur; l'admiration n'est due qu'à la Vertu. (1747: 106)

que el fausto no te preocupe, pues solo las almas pequeñas se postran delante de la grandeza; y la admiracion se debe solo á la virtud. (1781: 102 )

On a dans tous les tems négligé l'éducation des Filles [...] (1747: 55)

En todos los tiempos se ha tenido gran descuido en la educación de las niñas: (1781: 53)

Les vertus d'éclat ne font point le partage des Femmes mais bien les vertus simples \& paisibles. (1747: 63) 
Las virtudes brillantes no han sido tan propias de las mugeres como las virtudes sencillas, y pacificas. (1781:61)

II faut sur-tout [sic] éviter le caractere c'est toujours un mauvais personnage, \& rarement en faisant rire se fait-on estimer. (1747: 113)

Sobre todo se debe evitar el caracter bufon, que siempre es un mal personage, y rara vez haciendo uno reir, se hace estimar. (1781: 109)

Ayez de vrayes vertus, vous trouverez assez d'approbateurs. (1747: 63)

Ten verdaderas virtudes, que no faltará quien las apruebe. (1781:61)

Vous ne pouvez vous permettre qu'une seule vengeance, c'est celle de faire du bien à ceux qui vous ont offensée. (1747: 108)

Solo una venganza se te permite, que es hacer el bien á los que te han ofendido, [...] (1781: 104)

Employons donc le tems en connoissances utiles. (1747: 84)

Empleemos pues el trabajo en conocimientos utiles. (1781: 81)

Il faut craindre ces grands ébranlemens de l'ame, qui préparent l'ennuî \& le dégoût; (1747: 73)

Las grandes impresiones en el alma se deben temer, porque preparan el disgusto, y el fastidio; (1781: 70)

II ne faut qu'un service rendu à propos, ou une autre manière d'envisager les choses, pour vous les rendre plus attachés. (1747: 110)

Un servicio hecho á tiempo, ú otro modo de mirar las cosas, es bastante para volvertelos mas intimos. (1781: 105)

la santé\& l'innocence en font les vrayes sources. (1747: 73) 
la salud, y la inocencia son los verdaderos manantiales donde nace; (1781: 70)

Le silence convient toujours à une jeune personne: il y a de la modestie \& de la dignité à le garder. Vous jugez les autres,\& vous ne hazardez rien. (1747: 113)

El silencio conviene siempre á una joven, y es modestia, que parece muy bien el guardarle; y callando juzgas á los otros, y tú no arriesgas nada; (1781: 108)

En el plano morfosintáctico, como se ha dicho anteriormente, la técnica por excelencia es la articulación, que consiste introducir marcas lingüísticas de articulación que no aparecen en el texto original.

Las dos oraciones yuxtapuestas del texto original se han traducido como dos oraciones coordinadas copulativas con la introducción de la conjunción "y".

En este caso las dos oraciones independientes han pasado a ser traducidas como coordinadas copulativas mediante el nexo " $y$ ".

Junto a esta técnica nos encontramos con la reforma de oraciones que consiste en traducir una oración del texto original cambiando su tipología. En el primer ejemplo que se ofrece, las tres oraciones yuxtapuestas del texto original han sido traducidas por una oración condicional mediante la conjunción condicional "si".

En este segundo supuesto, la oración coordinada copulativa ha sido traducida por una oración de relativo en la que el antecedente es "adorno" y parece que este sea el que "tiene su lugar en las cosas graciosas"; sin embargo, la autora atribuye ese lugar a la limpieza y no al adorno.

En este último ejemplo, las dos primeras oraciones que son yuxtapuestas se han traducido como una oración compuesta formada por una proposición principal y otra subordinada causal al haber incluido la conjunción causal "pues".

Otra técnica profusamente aplicada en esta obra es la transposición, mediante la que se produce un cambio de categoría gramatical.

En este ejemplo, se ha traducido el verbo négliger por el sustantivo "descuido".

En este caso, dos sustantivos han sido sustituidos por dos adjetivos. El primero de ellos, el sustantivo "vertus" modificado por un sintagma preposicional "d'éclat" ha sido traducido por un sustantivo "virtud" modificado por un adjetivo "brillantes". Y el segundo, el sustantivo "partage" ha sido traducido por el adjetivo "propias". 
Otro ejemplo de transposición, lo constituye la sustitución del adverbio rarement por el sintagma nominal "rara vez" que encontramos en el siguiente ejemplo:

En cuanto a las modulaciones, tenemos que hacer distinción entre aquellas que se producen en el nivel morfosintáctico y las que corresponden al nivel léxico-semántico. De entre las que pertenecen al nivel morfosintáctico reproducimos dos de ellas, la primera de ellas supone la sustitución del verbo trouverez por su contrario negativado "no faltará". Junto a esta modulación se ha producido una transposición por el sustantivo approbateur ha sido sustituido por su definición "quien las apruebe" introducida por el pronombre de relativo "quien".

A continuación, vemos como la voz activa empleada en el texto original es traducida por la voz pasiva refleja en la traducción. En el texto original, Mme de Lambert le explica a su hija que ella solo puede permitirse vengarse haciendo el bien a los demás; sin embargo, en la traducción la atención se centra en la propia venganza permitida a Marie-Thérèse.

En cuanto a la modulación producida a nivel léxico-semántico, encontramos la siguiente en la que se ha producido el cambio del punto de vista por haber traducido el tems por "trabajo":

Descubrimos varias inversiones a lo largo del texto. En la primera de ellas, se han invertido los elementos de la oración; mientras que el texto original comienza advirtiendo que "hay que temer" algo, el texto traducido menciona en primer lugar "qué debe ser temido". Esta inversión lleva aparejada la transformación de la oración de relativo introducida por el pronombre "qui" en una oración subordinada causal introducida por la conjunción "porque".

En el segundo supuesto, la traductora ha optado por invertir el orden de los elementos de la oración en aras de una mayor claridad. Al mismo tiempo, podemos afirmar que se ha producido una modulación al traducir la expresión de la obligación II ne faut que en negativa, por el verbo "ser" y el adjetivo "bastante".

Hay que hacer referencia también a la explicitación mediante la que se expresa en la traducción elementos referidos mediante el pronombre "en" del texto original.

En este supuesto, la autora ha empleado la expresión "y callando" al inicio de la segunda oración, convirtiéndola en coordinada copulativa para dar continuidad y expresar el desarrollo de la idea implícita en el texto original.

Por último, analizaremos algunos supuestos de compresión lingüística.

En este caso, la traductora ha utilizado una única unidad léxica, el verbo "arreglar", que comparte la misma raíz semántica de la expresión francesa "mettez dans la règle". 
Mettez de la règle dans toutes vos vues \& dans toutes vos actions.

(1747: 74)

Arregla todas tus miras, y tus acciones: (1781: 71)

Si on vous a offensé, vous ne devez que du mépris, \&c'est une dette aisée à payer. (1747: 107)

Si te han ofendido, desprecialo, que es una deuda facil de pagar. (1781:

103)

Aquí vemos como la oración "vous ne devez que du mépris" ha sido traducida por una sola unidad lingüística "desprecialo" [sic].

En cuanto al plano léxico-semántico, las principales técnicas que encontramos en el texto son: la amplificación y la elisión. siguientes:

Respecto de la amplificación podemos poner como ejemplo los

Quel sera le principe de ces sentimens? (1747: 57$)$

¿Quál debe ser el principio de estos sentimientos? (1781: 54)

Quand on prescrit une conduite, [...] (1747: 57)

Quando se prescribe un modo de gobernarse [...] (1781: 55)

En el primero de ellos, se ha traducido el verbo "être" por "deber ser" expresando aquello que está implícito en el texto original. En el segundo caso, el sustantivo "conduite" ha sido traducido por "un modo de gobernarse".

Y en cuanto a la elisión, en el siguiente ejemplo observamos como la traductora ha obviado el complemento de nombre, "de malheurs", por estar explícito en la pregunta precedente. No obstante, queda la traducción algo incompleta al no expresar a qué se refiere ese "cierto numero" [sic].

De quel secours la Religion ne vous sera-t-elle pas contre les disgraces qui vous menacent? car un certain nombre de malheurs vous est destiné. (1747: 58)

¿De qué socorro no te será la Religión contra las desgracias que te amenazan? pues un cierto numero te está destinado. (1781: 55)

Desde el punto de vista pragmático-cultural, podemos resaltar que siempre que existe un equivalente cultural del topónimo o antropónimo referido en el texto original, la condesa de Lalaing opta por incluir la traducción. Sin embargo, en aquellos casos en los que no existe dicho equivalente se mantiene el nombre original francés. Por ejemplo, Germanicus 
ha sido traducido por Germanico, Anne de Bretagne por Ana de Bretagne, Charles-Quint por Carlos Quinto, Montaigne por Montagne [sic] (1781: 73), Pompée por Pompeyo, etc.

En cuanto a los errores, hay que destacar los leísmos, laísmos y el loísmo en que ha incurrido la traductora. La profusión de laísmos se debe a que el texto está dirigido a una mujer y los temas tratados son los del sexo femenino y tienen como protagonistas a otras mujeres.

que l'éducation des enfans leur est confiée dans la première jeunesse, tems où les impressions se font plus plus vives \& plus profondes. (1747: 56)

la educación de los hijos las está confiada en la niñez, tiempo en que las impresiones se hacen mas vivas, y mas profundas. (1781: 53)

El pronombre correcto es "les" y no "las" aunque se refiera a las mujeres. La traducción correcta sería: "la educación de los hijos les está confiada en la niñez"

Peu de gens savent l'estimer sélon sa juste valeur. (1747: 79)

Pocas gentes saben estimarle segun su justo valor. (1781: 76)

Se ha utilizado el pronombre "le" en lugar de "lo". La traducción correcta sería: "Pocas gentes saben estimarlo según su justo valor".

Songez que vous ne devez qu'au hazard l'extrême difference qu'il y a de vous à eux: ne leur faites point sentir leur état : n'appesantissez point leur peine. (1747: 114)

Piensa tambien, que solo debes á la casualidad la extrema diferencia que hay de ti á ellos, y no los hagas sentir su estado: no hagas mas pesado su trabajo, [...] (1781: 110)

Se ha utilizado el pronombre "los" cuando lo correcto, en este caso, es "les". De esta forma la traducción sería: "y no les hagas sentir su estado".

Además de los laísmos, son muy frecuentes las omisiones que, a veces, afectan a fragmentos cortos o a una parte de una oración y, otras veces, a párrafos completos.

si vous avez de la beauté, il ne faut pas user le goût du public en vous montrant toujours; il faut encore être plus retenue, si vous êtes sans graces: d'ailleurs le grand usage des spectacles affoiblit le goût. (1747: 72) 
Si tienes hermosura, no abuses del gusto del público, presentandote continuamente; es menester ser mas prudente: y advierte además, de que la gran freqüencia á los expectáculos minora el gusto de verlos. (1781:69)

Se ha omitido la traducción de la proposición condicional "si vous êtes sans graces". Esta omisión afecta a la traducción pues Mme de Lambert recomienda a su hija que cuando no se es agraciada físicamente hay que ser más prudente; sin embargo, en la traducción se dice que es "menester ser más prudente" con independencia de las gracias que se posean. Una completa traducción del fragmento podría ser: "Si tienes hermosura, no abuses del gusto del público presentándote continuamente; hay que ser más contenida aun si careces de gracias".

Otra omisión descubierta en la traducción es la del siguiente párrafo:

les Auteurs Italiens sont peu châtiés: il regne dans leurs ouvrages un jeu de mots, une imagination sans règle, qui s'oppose à la justesse de l'esprit. (1747: 81)

Una posible traducción del mismo podría ser: "Los autores italianos son poco correctos. En sus obras reina un juego de palabras, una imaginación desordenada que se opone a la rectitud".

También se han detectado varios errores que consisten en falsos sentidos. A continuación, se exponen algunos ejemplos.

II faut donc ménager ses goûts; nous ne tenons à la vie que par eux. (1747: 72)

Es preciso reglar tus gustos, pues no tenemos la vida solo para ellos. (1781: 70)

La expresión "tenir à", según el Diccionnaire Le Petit Robert, significa: "Y être attaché par un sentiment durable". La traductora lo ha traducido por "tener" pero no lleva consigo el sentido de posesión como aparece en la traducción. Este fragmento podría traducirse por: "Es preciso reglar sus gustos; solo apreciamos la vida por ellos".

Examinez votre caractere, \& mettez à profit vos défauts; il n'y en a point qui ne tienne à quelques vertus, \& qui ne les favorise. (1747: 93)

Exâmina tu caracter, y saca partido de tus defectos: nadie hay que no tenga alguna virtud que le favorezca. (1781: 89) 
En este fragmento, la traductora ha entendido que son las virtudes las que favorecen al que las posee, sin embargo, el pronombre "qui" nos dice que es el poseedor de esas virtudes el que las favorece a ellas en el sentido del DRAE de 1780 de "patrocinar, ayudar, amparar,...". Una posible traducción sería: "Examina tu carácter y saca partido de tus defectos: nadie hay que no aprecie ciertas virtudes y que no las favorezca."

En el ejemplo que se muestra a continuación, llama la atención la aparente falta de concordancia en la traducción del presentativo "ce sont les sentimens qui..." ya que emplea el pronombre de relativo "quien" en singular cuando se refiere a los "sentimens".

ce sont les sentimens qui forment le caractere, qui conduisent l'esprit, qui gouvernent la volonté, qui répondent de la réalité (1747: 56)

los sentimientos son quien forman el caracter, quien dirige el espiritu, quien gobierna la voluntad, quien responde de la realidad, [...] (1781: 54)

Por último, la traducción que se realiza de la locución prepositiva "voici" es muy literal. Efectivamente, ha sido traducida por "ve aqui" que corresponde literalmente a los elementos que la conforman, el imperativo del verbo "voir" y la preposición "ici". El diccionario Le Littré nos dice que: "Il annonce qu'on va exposer, détailler quelque chose". Por esta razón, se propone la siguiente traducción que es menos literal y más acorde con su significado: "He aquí" o "estos son".

voici les remedes pour en arrêter le progrès. (1747: 95)

ve aqui los remedios para detener el progreso. (1781: 92)

\section{CONCLUSIONES}

Influyente "salonnière", hasta el punto de ser conocido su salón como la antecámara de la Académie Française, la figura de Mme de Lambert está enmarcada en la literatura francesa entre el Clasicismo y la llustración. Su obra refleja cierta influencia del preciosismo, pero a la vez se encuentra abierta a las nuevas ideas propiciadas por los avances científicos de la época. La sujeción a las conveniencias sociales y su preocupación pedagógica son dos de las principales características de su obra. En el Avis d'une mère à sa fille, opúsculo destinado a su hija, vierte sus reflexiones más íntimas mostrándose como una madre sin debilidades, empleando un estilo sencillo y natural, salpicado de brillantes máximas extraídas de su propia vivencia y del pensamiento de autores clásicos. A pesar del reconocimiento y la admiración de los que gozó en Francia, fue poco conocida en España. La 
primera traducción autorizada que se hizo de su obra corre a cargo de la condesa de Lalaing, quien quiso obsequiar a la Princesa de Asturias, $D^{\underline{a}}$ Luisa de Borbón con "las Obras de la Marquesa de Lambert (Señora muy recomendable por todas sus circunstancias)". En su traducción, la condesa reescribe el texto manteniéndose fiel al original utilizando los elementos léxicos y discursivos propios de la lengua española, a pesar de leves omisiones. Con esta versión, la obra de la marquesa de Lambert se abre paso en nuestro país, donde en fechas más recientes, la autora ha vuelto a despertar el interés que merece "cette personne de mérite, si délicate à la fois et si bien pensante, et qui fit de ses qualités et de sa fortune un si noble usage" como dijo de ella Sainte-Beuve.

\section{REFERENCIAS BIBLIOGRÁFICAS}

CERDA Y VerA, María Cayetana (de la), Condesa de Lalaing, Obras de la Marquesa de Lambert traducidas del Francés. Madrid: Manuel Martín, 1781.

Delorme, Suzanne, "Le Salon de la Marquise de Lambert, berceau de l'Encyclopédie". En: Revue d'histoire des sciences, 3, 1951, pp. 223227.

Henault, C.J.F., Mémoires du président Hénault. Genève: Slatkine reprints, 1971. Disponible en línea en: https://goo.gl/cz1341 [Fecha de la consulta: 29 de noviembre de 2016].

LAmOthe-Fenelon, Francisco de Salignac (de), Éducation des filles. Paris: Pierre Aubouin, P. Emery et C. Clousier, 1687. Disponible en línea en: https://goo.gl/nNKt49 [Fecha de la consulta: 3 de marzo 2017].

Malebranche, Nicolas, Oeuvres de Malebranche, T.III, Recherche de la vérité. Paris: Entier et Cie, Libraires-Éditeurs, 1871. Disponible en línea en: https://goo.gl/oJrFaU [Fecha de la consulta: 15 de enero de 2017].

Marguenat De Courcelles, Anne Thérèse (de): CEuvres de Madame la marquise de Lambert, rassemblées pour la première fois. Lausanne: Bousquet \& Compagnie, 1747.

SAINTE-BeuVE, Charles Augustin: Causeries du Lundi, quatrième édition revue et corrigée, tome quatrième. Paris: Garnier Frères, Librairie, 1852, pp. 217-239.

ZIMMERMANN, J. P.: "La morale laïque au commencement du XVIIIe siècle: Madame de Lambert". En: Revue d'histoire littéraire de la France, 3, 1917, pp. 42-64. Disponible en línea:

http://gallica.bnf.fr/ark:/12148/bpt6k5767355h/f45.vertical [Fecha de la consulta: 4 de julio de 2017]. 
RECURSOS ELECTRÓNICOS:

Académie Française. Disponible en línea en: http://www.academiefrancaise.fr [fecha de consulta: 12 de junio de 2017].

Bibliothèque nationale de France. Disponible en línea en: http://gallica.bnf.fr/ [Fecha de consulta: marzo de 2017].

Centre national de ressources textuelles et lexicales. Disponible en línea en: http://www.cnrtl.fr/definition/ [Fecha de consulta: 12 de marzo de 2017]

LITTRE, Émile: Dictionnaire Le Littré Disponible en línea en: http://www.littre.org/ [Fecha de consulta: 2 de mayo de 2017].

RAE. Consultas. Disponible en línea en: http://www.rae.es/consultas/uso-delos-pronombres-los-las-les-leismo-laismo-loismo. [Fecha de consulta: 2 de mayo de 2017]

Diccionario de la lengua española. Madrid: Espasa Calpe, 2001.

Diccionario de la lengua castellana. Madrid: Joaquín Ibarra, 1780. Disponible en línea en: https://goo.gl/Zixa63 [Fecha de consulta: 5 de mayo de 2017].

Ortografía española. Madrid: Real Academia Española, 1741. Disponible en línea en:

http://www.rae.es/sites/default/files/Ortografia_RAE_1741_reducida. pdf [Fecha de consulta:7 de junio de 2017].

Gramática de la lengua castellana. Madrid: Joachim de Ibarra, 1771. Disponible en línea en:

http://www.rae.es/sites/default/files/Gramatica_RAE_1771_reducida. pdf [Fecha de consulta: 7 de junio de 2017]. 